\title{
Las razones de por qué Chile debe detener la importación del abejorro comercial Bombus terrestris (Linnaeus) y comenzar a controlarlo
}

\section{The reasons why Chile should stop importing commercial bumblebee Bombus terrestris (Linnaeus) and to start controlling it}

\author{
Cecilia Smith-Ramírez ${ }^{1,2,3^{*}}$, Lorena Vieli ${ }^{4}$, Rodrigo M. Barahona-Segovia ${ }^{5,6}$, José Montalva ${ }^{7}$, Franco \\ Cianferoni ${ }^{8}$, Luisa Ruz ${ }^{9}$, Francisco E. Fontúrbel ${ }^{9}$, Carlos E. Valdivia ${ }^{1}$, Rodrigo Medel ${ }^{8}$, Aníbal \\ Pauchard ${ }^{10,2}$, Juan L. Celis-Diez ${ }^{11}$, Vladimir Riesco ${ }^{3}$, Víctor Monzón ${ }^{12}$, Felipe Vivallo ${ }^{13}$ \& Miguel \\ NEIRA ${ }^{14}$
}

${ }^{1}$ Dpto. de Ciencias Biológicas y Biodiversidad, Universidad de Los Lagos, Avda. Fuchslocher 1305, Osorno, Chile. ${ }^{2}$ Instituto de Ecología y Biodiversidad-Chile (IEB), Las Palmeras 3425, Ñuñoa, Santiago, Chile.

${ }^{3}$ Inst. de Conservación, Biodiversidad y Territorio, Universidad Austral de Chile, Isla Teja s/n, Valdivia, Chile.

${ }^{4}$ Departamento de Ciencias Agronómicas y Recursos Naturales, Universidad de La Frontera, Avda. Francisco Salazar 01145 , Temuco, Chile

${ }^{5}$ Laboratorio de Ecología de Ambientes Fragmentados, Dpto. de Ciencias Biológicas Animales, Fac. de Ciencias Veterinarias y Pecuarias, Universidad de Chile, Santa Rosa 11735, La Pintana, Santiago, Chile.

${ }^{6}$ Centro de Estudios en Ecología Espacial y Medio Ambiente - Ecogeografía, Av. José Miguel Claro 2550, Providencia, Santiago, Chile.

${ }^{7}$ Department of Biology, East Central University, 146 Physical and Environmental Sciences Building, Ada, OK.

${ }^{8}$ Fac. de Ciencias, Universidad de Chile, Las Palmeras 3425, Ñuñoa, Santiago, Chile.

9Instituto de Biología, Pontificia Universidad Católica de Valparaíso, Av. Universidad N³30, Valparaíso, Chile.

${ }^{10} \mathrm{Lab}$. Invasiones Biológicas, Fac. de Ciencias Forestales, Universidad de Concepción, Casilla 160-C, Concepción, Chile

${ }_{11}^{11}$ Pontificia Universidad Católica de Valparaíso, Escuela de Agronomía, Casilla 4-D, 2260000 Quillota, Chile.

${ }^{12}$ Lab. Ecología de Abejas, Depto. Biología y Química. Facultad de Ciencias Básicas. Universidad Católica del Maule. Avda. San Miguel 3605. Talca, Chile.

${ }^{13}$ HYMN Laboratório de Hymenoptera, Museu Nacional/ Universidade Federal do Rio de Janeiro, Quinta da Boa Vista, São Cristóvão 20940-040, Rio de Janeiro, Brasil.

${ }^{14}$ Facultad de Ciencias Agrarias, Universidad Austral de Chile, Isla Teja s/n, Valdivia, Chile.

*E-mail: ceciliasmithramirez@gmail.com

\begin{abstract}
RESUMEN
La crisis mundial de polinización no solo es producida por la pérdida de polinizadores, sino también por su homogeneización, ambos procesos causados por el ser humano. En este documento analizamos los impactos ecológicos que justifican la prohibición de nuevos ingresos del abejorro comercial Bombus terrestris a Chile. Esta especie es altamente invasiva y ha sido prohibida en otros países, pero en Chile se permite su ingreso desde 1997. Miles de colonias y reinas fertilizadas ingresan al país cada año y se expanden por el resto de Sudamérica. En este artículo entregamos once razones para detener los ingresos de $B$. terrestris a Chile y comenzar a controlarlo. Justificamos estas razones en base al trabajo realizado tanto en el país como fuera de él. Al mismo tiempo, a través de las conclusiones, mencionamos cuáles deberían ser los pasos a seguir respecto a la prohibición de ingreso de B. terrestris a Chile.
\end{abstract}

Palabras clave: Apis mellifera, Bombus dahlbomii, enfermedades de abejas, insectos invasores, polinizadores.

\begin{abstract}
The global pollination crisis is not only produced by loss of pollinators but also by their homogenization, both processes caused by humans. This crisis impacts the persistence of many native plants species worldwide. In this document we analyze the ecological impacts that warrant the prohibition of new income of commercial bumblebee, Bombus terrestris to Chile. This species is highly invasive, it has been banned in other countries, but in Chile its entry is allowed since 1997. Thousands of colonies and fertilized queens entered the country every year and expand throughout the rest of South America. We give eleven reasons to stop $B$. terrestris entry to Chile and start controlling it. We justify these reasons based on work done both in the country and outside of it. At the same time, through the conclusions, we mentioned what should be the steps to followed regarding the prohibition $B$. terrestris entry to Chile.
\end{abstract}

KEYwords: Apis mellifera, Bombus dahlbomii, bee diseases, invasive insects, pollinators. 
Por qué Chile debe detener la importación de Bombus terrestris: Smith-RAmírez, C. ET AL.

\section{INTRODUCCIÓN}

La crisis mundial de la polinización no solo es producida por la pérdida de polinizadores sino también por su homogeneización, la cual es causada principalmente por la invasión de himenópteros generalistas introducidas accidental o intencionalmente (Potts et al. 2016). El abejorro Bombus terrestris (Apidae), ha sido introducido por razones comerciales en distintas partes del mundo como agente polinizador (Dafni et al. 2010), donde generalmente se ha convertido en una especie invasora con importantes efectos sobre la persistencia de especies nativas y conservación de la biodiversidad (Hingston \& MacQuillan 1998; Matsumara et al. 2004; Hingston 2006; Inoue et al. 2008; Morales et al. 2013; Schmid-Hempel et al. 2014). En Chile, esta especie está generando un problema ambiental y probablemente económico creciente, existiendo peticiones para cesar su importación y búsqueda de formas de control de sus poblaciones. Sin embargo, aún está permitida la importación de $B$. terrestris en Chile. En este documento analizamos los impactos ecológicos que ameritan la inmediata prohibición de nuevos ingresos de ejemplares de esta especie.

Proceso de InTROducción E InVASIÓN

En el año 1997 se ingresaron por primera vez a Chile, con permiso del Servicio Agrícola y Ganadero (SAG), 404 colonias del abejorro comercial B. terrestris (Hymenoptera: Apidae; SAG 2016). Parte de estas colonias se usaron en un proyecto financiado por la Fundación para la Innovación Agraria (FIA), ejecutado por la Pontificia Universidad Católica de Valparaíso para realizar ensayos sobre la eficiencia en la polinización del tomate (Schmid-Hempel et al. 2014). Los ensayos fueron realizados tanto en invernadero como al aire libre. Debido a que los resultados de estos ensayos fueron positivos, a partir de 1998, se continuaron ingresando colonias de la especie en forma paulatina y luego de forma masiva desde el 2006 (Estay 2007), extendiéndose su uso a arándanos y cerezos, entre otros cultivos.

Desde el ingreso de $B$. terrestris a Chile, este se ha extendido espontáneamente hasta la costa Atlántica del cono sur de Sudamérica (Aizen et al. 2018) y al altiplano chilenoboliviano (Montalva et al. 2017). Hasta el año 2016, Chile había ingresado un promedio anual de 16.650 colonias y 95.670 reinas (ya sea con oviposturas o fertilizadas), sumando, desde su introducción, un total aproximado de 335.000 colonias y 860.000 reinas (SAG 2016, 2017; Aizen et al. 2018). Cada colonia comercial contiene aproximadamente 50 obreras al momento de ser dispuestas en los campos/invernaderos, pudiendo posteriormente aumentar hasta 200 (Velthius \& van Doorn 2006). Si bien no se comercializan machos, las obreras por partenogénesis pueden dar origen a princesas y estas a machos. El ingreso al país ocurre por Aduanas en el Aeropuerto Internacional de
Santiago (SCL), previa inspección externa de una muestra de abejorros realizada por el SAG (SAG 2016). Además, desde el año 2001, en Chile se han estado produciendo colonias de $B$. terrestris a través de la compra de reinas fertilizadas (SAG 2016) que son criadas por la empresa BioCruz. Cómo no hay registros oficiales y públicos de las ventas nacionales, se desconoce la cantidad exacta de abejorros comerciales que están siendo liberados al ambiente anualmente.

Las empresas chilenas intermediarias entre productores y compañías extranjeras, han comprado y compran B. terrestris a varias empresas, entre ellas Koppert (de Holanda) y Biobest (de Bélgica). En abril de este año (2018), Biobest reconoció en un diario belga que no dimensionó el impacto que las importaciones de $B$. terrestris tendrían en los ecosistemas de Latinoamérica (ver antecedentes en el repositorio figshare: https://doi.org/10.6084/m9.figshare.6291599, donde se incluye traducción del flamenco). Días después, Biobest reconoció en su página web que la decisión de autorizar importaciones de $B$. terrestris a Chile fue un error, pero ellos no detendrán las importaciones, ya que hacerlo no ayudaría a la recuperación del abejorro nativo, B. dahlbomii (GuérinMéneville) (también disponible en el repositorio figshare).

Impactos Ecológicos Directos de la EsPecie

Bombus terrestris es considerado un insecto-plaga (Kenis et al. 2008; Dafni et al. 2010) debido a su alta tasa de reproducción, su gran capacidad de adaptarse a distintos ambientes, y en el caso de los individuos criados comercialmente y en hacinamiento, a su alta prevalencia de enfermedades (Murray et al. 2013). Donde B. terrestris ha sido introducido, se ha reportado la declinación en abundancia de abejorros nativos (en Japón), de la abeja melífera Apis mellifera (Linnaeus) y eventualmente de abejas nativas de vida solitaria (Dafni \& Schmida 1996; Inari et al. 2005; Morales et al. 2013; Smith-Ramírez et al. 2014). En el caso de Chile, específicamente en Chiloé, B. terrestris ha producido la declinación de hasta un $99,4 \%$ de la abundancia relativa del abejorro nativo $B$. dahlbomii, y de cerca de un $40 \%$ de la abeja melífera asilvestrada (Smith-Ramírez et al. 2014). Adicionalmente, durante el forrajeo B. terrestris daña muchas especies de plantas tanto silvestres como de cultivos (Kendall \& Smith 1976; Kenta et al. 2007; Aouarsadli et al. 2008; Sáez et al. 2017), disminuyendo además la cantidad de néctar disponible para otras abejas, insectos y aves nectarívoras (Graham 2015; Valdivia et al. 2016; Sáez et al. 2017) que podrían actuar como agentes polinizadores. No obstante los efectos negativos mencionados, estudios de monitoreo interanual (tres años) en un ecosistema de altura de Chile central no han revelado una asociación clara de $B$. terrestris con la fauna de polinizadores; aunque se desconoce cuál era la diversidad y abundancia de estos antes de la llegada del abejorro comercial (Esterio et al. 2013). Por otra parte, se ha reportado que la especie invasora favorece la reproducción del berro cordillerano Erythranthe lutea 
(Douglas ex Benth; G.L.Nesom y N.S.Fraga), una especie nativa de Chile (Medel et al. 2018).

\section{Patógenos CO-INVASORES}

Las enfermedades que el abejorro comercial porta son transmisibles a otros abejorros de su misma especie, a sus congéneres nativos no comerciados, a abejas melíferas y otros Hymenoptera, pudiendo afectar a abejas solitarias (Dafni \& Schmida 1996; Genersch et al. 2006; Singh et al. 2010; Meeus et al. 2011; Murray et al. 2013). Estas enfermedades no son detectadas por la inspección externa que realiza el SAG, debido a que son patógenos internos (Graystock et al. 2013). Cabe mencionar, que al ingresar los abejorros a cada país comprador, van acompañados por una carta de la empresa que los vende diciendo que están libres de una serie de enfermedades; según protocolo sanitario internacional, la veracidad de esta carta es avalada por el país de origen de la importación.

Dados los impactos negativos causados por $B$. terrestris y la desconfianza en el contenido de las cartas antes mencionadas, Graystock et al. (2013) realizaron un estudio en base a ADN de las enfermedades presentes en colonias comerciales. Los autores compraron abejorros comerciales a tres empresas europeas: Biobest, Koppert y Syngenta (Peter Graystock com. pers., ruc 181000582 Fiscalía, Chile). Los autores de este estudio encontraron que el $70 \%$ de los abejorros estudiados, y el alimento con que los proveían dichas empresas, venían infectados por los parásitos que los certificados sanitarios decían que estaban libres; y además, contenían otras enfermedades transmisibles. Los patógenos detectados fueron Nosema bombi (Fantham \& Porter) y Nosema apis (Zander), ambos con una baja incidencia, y Apicystis, DWV (virus de deformidad alar), Ascosphaera y Crithidia bombi, todos ellos en alta frecuencia. Adicionalmente, también fueron detectados otros agentes patógenos en el alimento con que iban suplementadas las colonias. Poco después del estudio de Graystock et al. (2013), Bombus impatiens (Cresson), otra especie producida a escala comercial y vendida en Norteamérica, fue analizada en busca de los patógenos que sus certificados especificaban como ausentes. Los resultados obtenidos indicaron la presencia de patógenos, incluyendo aquellos que producen el colapso de las colmenas de abejas melíferas (Sachman-Ruiz et al. 2015). A esto se suman resultados similares encontrados por Murray et al. (2013) en $B$. terrestris comerciales liberados en cultivos en Europa.

ACCIONES PARA DISMINUIR EL IMPACTO

Actualmente, se sabe que los procesos de invasiones biológicas son uno de los mayores desafíos en la conservación de la biodiversidad y uno de los motores de extinción de especies (Simberloff et al. 2013). Muchas veces existe una brecha entre la divulgación del quehacer científico y la toma de decisiones políticas, por lo cual, nuestra intención es sintetizar y hacer disponible los argumentos científicos que justifican el cese inmediato de las importaciones de B. terrestris a Chile. Desde mediados del 2016 este cese está siendo solicitado al SAG por científicos argentinos y chilenos (Aizen et al. 2018). Es de notar, que si bien Argentina prohibió el ingreso de $B$. terrestris, este arribó espontáneamente proveniente de Chile, constituyéndose en un problema ambiental para este país (Aizen et al. 2018). Incluso $B$. terrestris potencia la expansión de plantas extremadamente invasoras como Cytisus scoparius (L.) Link, no sólo en Argentina (Morales et al. 2017) sino que también en Chile. Un caso similar ocurrió cuando EEUU supo del ingreso de $B$. terrestris a Norteamérica proveniente de México (temporada 1995-6) los cuales portaban Nosema bombi. En esa ocasión EEUU solicitó a México, en base a tratados sanitarios internacionales, que eliminara sus colonias, lo cual se realizó (Winter et al. 2006). En el caso de $B$. terrestris, la responsabilidad de las autoridades chilenas (representadas por el SAG) tiene repercusión internacional significativa. Además del caso de la invasión en Argentina (Aizen et al. 2018), un estudio reciente (Acosta 2015) mostró que B. terrestris tiene una elevada probabilidad de invadir gran parte de la costa atlántica llegando hasta el centro-sur de Brasil, pasando por Uruguay. Bajo este escenario, Chile estaría actuando como una megafuente permanente de abejorros invasores, con un potencial significativo de ocasionar una crisis de biodiversidad a nivel Latinoamericano, y afectar cultivos por el fenómeno llamado sobre-polinización (se explica más abajo).

Por otro lado, existe una demanda por parte de empresas chilenas por el servicio de polinización de $B$. terrestris, principalmente para tomate de invernadero. Este servicio les permite a los empresarios disminuir costos de mano de obra e incrementar su ganancia. Aproximadamente el 80\% de las colonias que ingresan al país son usadas en invernadero para la producción de tomate (M. Gallardo -funcionario del SAG- com. pers.). En invernadero se produce el 17\% de la producción de tomate del país, el cual el año 2007 se usaba mayormente para pulpa, y apenas un $2 \%$ para consumo fresco (http://www.odepa.cl/estadisticas/productivas/). Estadísticas más recientes de ODEPA (2013), señalan que el tomate para consumo fresco sería aproximadamente el $20 \%$ de la producción de invernadero, superando las 1000 ha. Antes de usarse B. terrestris para producción de tomate en invernadero, se usaban hormonas o un método de vibración que hacía que el polen se desprendiera de las anteras y alcanzara el estigma de las flores. En las chacras simplemente se dejaba o deja el cultivo libre a la visita de los polinizadores que hubiese.

La mayor producción de tomate en invernadero en Chile proviene de aquellos que se consumen en invierno, los cuales se cultivan en el valle de Azapa (extremo norte de Chile). En estos invernaderos se cultivan variedades de alto rendimiento, que se ha traducido en una leve disminución 
Por qué Chile debe detener la importación de Bombus terrestris: Smith-RAmírez, C. ET AL.

de su precio para el consumidor, en desmedro de la producción en Chile central (ODEPA 2013). Es de notar que los estudios de ODEPA (Oficina de Estudios y Políticas Agrarias del Ministerio de Agricultura) no mencionan como factor de la leve caída del precio de tomate, al cambio de uso de hormonas y vibración por el uso de $B$. terrestris como polinizador. Es decir, es posible postular que el dejar de usar $B$. terrestris y volver a los antiguos métodos de polinización no debiera traducirse en mayores costos para el consumidor.

En la Región de Arica y Parinacota (donde se encuentra el valle de Azapa) individuos escapados de B. terrestris, están en contacto con especies nativas de Hymenoptera, entre ellas el abejorro del altiplano, Bombus funebris (Smith) y abejas carpinteras del género Xylocopa (Latreille), con quienes $B$. terrestris también comparte enfermedades (Tehel et al. 2016). El efecto que $B$. terrestris tiene no sólo sobre las especies nativas del género Bombus, sino sobre otras abejas es especialmente negativo, ya que se ha demostrado que especies del género Xylocopa, son excelentes polinizadores de tomate (Hogendoorn et al. 2000). No hay estudios respecto a la efectividad de $B$. funebris como polinizador de cultivos, ni de las Xylocopa u otras abejas nativas del país, excepto, para B. dahlbomii. Este último, demostró ser, por lo demás, un buen polinizador de tomate (Estay 2007). Por otra parte, desconocemos estudios productivos sobre el uso de invernaderos semi abiertos que permitan la entrada de polinizadores nativos. En Chile, además de los Bombus existen varias especies de polinizadores nativos que producen el zumbido necesario para el polen se desprenda de las anteras de las flores de tomate (polinización especializada llamada polinización por zumbido). Los géneros de polinizadores nativos que producen polinización por zumbido son Centris, Caupolicana, Diphaglossa, Cadeguala, Alloscirtetica, entre otros.

A continuación enumeramos y justificamos once razones por las cuales Chile debe detener la importación de $B$. terrestris, y comenzar a controlarlo. Los puntos no se enumeran en orden de importancia.

1.- Peligro de ingreso de nuevos Genotipos de B. TERRESTRIS, lo cual hace aún más exitoso su establecimiento en los distintos climas de Chile y de Sudamérica en general. Investigaciones en desarrollo (Cianferoni \& Poulin, en preparación) han mostrado una alta estructuración y diferenciación genética en poblaciones asilvestradas de B. terrestris, especialmente en las localidades más al norte y centro de la distribución actual de la especie en Chile. Teniendo en cuenta que la primera introducción de $B$. terrestris fue tan solo hace 20 años (liberado desde Arica a Los Ángeles), esta gran diferenciación y estructuración genética estarían dando cuenta del gran potencial colonizador de la especie y del potencial adaptativo a nuevos ambientes.
2.- Peligro de introducción de nuevas Cepas de ENFERMEDADES Y RENOVACIÓNGENÉTICADEENFERMEDADES presentes en Chile y Argentina. Schmid-Hempel et al. (2014) mostraron que la diversidad genética del parásito de abejorros Crithidia bombi ha ido en aumento en los abejorros comerciales presentes en Chile, es decir, se ha introducido una mayor cantidad de variantes genéticas en el tiempo. Entre seis y siete años después del primer análisis genético de este parásito (2004 respecto 2011-2012), se encontró que había aumentado su heterocigosidad y que su población estaba estructurada por aislamiento por distancia, de la misma forma en que lo estaba su hospedero $B$. terrestris.

3.- Problemas de SOBRe-POLINIZACión PRODUCido POR SOBREABUNDANCIA DEL POLINIZADOR. Las abundancias de $B$. terrestris son extremadamente altas y el ingreso de miles de reinas y/o colonias cada año contribuyen a aumentarlas aún más. Los problemas en cultivos producidos por el abejorro comercial son causados por su superabundancia, la cual no es controlada en la naturaleza (como ocurre con las especies invasoras). Esta superabundancia ha llevado, por ejemplo, a que miembros de comunidades mapuches no siembren habas en sus huertos, debido a que $B$. terrestris destruye las flores (N. Chocorí, com. pers. miembro de la Red Huerta Mapuche, Kum Ñi Tukukam). Además, ha sido encontrado que dañan la producción de frambuesa por romper los estilos de la flor (Sáez et al. 2014). En este cultivo se ha encontrado un promedio de aproximadamente 300 visitas/flor/día. En Europa se ha reportado que por sobre-abundancia dañan la producción de frutillas y podría afectar la producción del tomate cherry (Lieten 1993), pudiendo también afectar la producción de porotos (Kendall \& Smith 1976). La sobre polinización por abejorros ha sido señalado que puede también dañar la producción de Rosáceas como el damascos, y de otras especies como almendras (www.beesource. com, www.pollination.ca), aunque no hay estudios científicos al respecto.

4.- El uso de colmenas comerciales en Cultivos NO SIEMPRE RESULTA EN UN INCREMENTO DE LOS RENDIMIENTOS. Se ha reportado en otras partes del mundo, que $B$. terrestris puede aumentar la producción de frutos y semillas en varios cultivos, tanto en invernadero (van Raventijn \& Steen 1991; Abak et al. 1995; Dasgan et al. 2004) como en el campo (Zisovich et al. 2012). Sin embargo, en Chile debido al exitoso asilvestramiento de $B$. terrestris, la colocación de colmenas en cultivos puede ser redundante y no se justifica a partir de estudios (Aizen et al. 2018 reanalizado de Vieli et al. 2016, para el caso de arándano en Chile y de frutilla en el 
SW de España Trillo et al. 2018) e incluso, como se dijo, puede generar bajas en los rendimientos de cultivos como frambuesa (Sáez et al. 2017) y frutilla (Lieten 1993). Es necesario realizar estudios para establecer cuando es necesario adicionar colmenas comerciales, además de las asilvestradas, para la producción de cultivos en campo. No se debe suponer que mientras más polinizadores habrá una mayor producción, lo cual es una falacia. Adicionar colmenas "por si acaso", es un error común en el cual incurren algunos asesores agrarios. También es un error basarse en conocimiento de estudios al aire libre realizados en Europa, donde no hay sobre abundancias de $B$. terrestris.

5.- $\quad$ Robo DE NÉCTAR EN PLANTAS NATIVAS Y POSIBLE IMPACTO EN SU REPRODUCCIÓN. B. terrestris rompe la base del cáliz de las flores (acción llamada robo) de plantas que tienen flores alargadas, estrechas y productoras de néctar. Haciendo lo mismo en flores que no se han abierto aún, así como en flores de leguminosas, aunque no presenten flores alargadas. $\mathrm{Al}$ acceder al néctar por la base de la flor, no les brinda el servicio de polinización, y además provoca reducción en la producción de néctar (Graham 2015, Saéz et al. 2017 para frambuesa), afectando la polinización legítima de estas. Por ejemplo, el arbusto nativo Fuchsia magellanica (Lam.), presenta flores que eran polinizadas mayormente por el picaflor, pero después que ocurre robo de néctar por $B$. terrestris, ya no son visitadas por esta ave (Valdivia et al. 2016). Dohzono et al. (2008), detectaron en flora de Japón, que el robo de néctar por $B$. terrestris se traduce en menos producción de semillas. Lo anterior no ha sido detectado en F. magellanica, y los pocos los estudios realizados no han incluido la calidad de semillas producidas por autopolinización respecto a polinización cruzada. Además, de robar en las flores de este arbusto, se ha cuantificado esta acción en Embothrium coccineum y Sarmienta repens (Valdivia et al. 2018 y E. Ramíres datos sin publicar).

6.- EN LA MEDida QUe AUMenta la CANTIDAD DE $B$. TERRESTRIS EN EL PAís, DISMINUYE LA POSIBILIDAD DE recuperar de la categoría En Peligro de Extinción Al ABEjorRo nativo $B$. DAHLBOMII. Existe no sólo un proceso de transmisión de enfermedades entre ambos abejorros, generando una dinámica de parásitohospedero de segundo orden (Smith-Ramírez et al. 2014; Smith-Ramírez et al. en preparación), sino que esta dinámica es alternada entre años, es decir, un año es muy abundante uno y menos abundante el otro, y al año siguiente se presenta la dinámica inversa. Los altos y bajos en abundancia se producen por la dinámica parásito-hospedero, pero la alternación en abundancia entre años entre las especies, que probablemente se debe a la competencia por recursos. Estudios realizados en Japón muestran que la disminución del abejorro comercial está asociada a la recuperación de sus especies nativas de abejorros, mediada al parecer por la liberación de recursos (Nagamitsu et al. 2010), o en una segunda interpretación, además por un menor contacto con individuos transmisores de enfermedades. Es decir, la recuperación de la especie nativa está mediada por la disminución de la especie invasora. Si bien el control de B. terrestris es posible (como se menciona más adelante), mientras se fomente el aumento en abundancia de la especie invasora, será más difícil recuperar la población de la especie nativa.

7.- Amenaza a la producción melífera. Cómo se mencionó más arriba varias son las enfermedades que $B$. terrestris comparte con la abeja melífera. En el caso del estudio a largo plazo que se realiza en el norte de Chiloé, se mostró que la llegada de B. terrestris también afectó la población de abejas melíferas asilvestradas (Smith-Ramírez et al. 2014). La continua introducción de nuevos patógenos de procedencia genética europea al interior de $B$. terrestris, agrava los problemas actuales de prevalencia de patógenos tanto internos como externos de la abeja melífera, produciendo efectos negativos en una industria que el país a tratado de promover con diversos incentivos, y de la cual dependen muchas familias.

8.- AMENAZA DE EXPANSIÓN A OTROS PAíses SUDAMERICANOS y AFECTAR a otras ESPECIES De Bombus. Sudamérica cuenta con un total de 42 especies de Bombus (Abrahamovic \& Díaz 2002), de los cuales 14 habitan en Perú (Rasmussen 2003), 12 en Bolivia y 8 en Argentina (excluyendo a los exóticos; Abrahamovic \& Díaz 2002). Modelos de nichos recientes de $B$. terrestris pronostican que la especie se expandirá a Uruguay, Paraguay e incluso hasta el centro-sur de Brasil por la costa atlántica (Acosta 2015; Acosta et al. 2016), mientras que por el Pacífico amenaza con expandirse por Perú y Bolivia (Montalva et al. 2017). De hecho existe un registro de $B$. terrestris por confirmar en el Parque Nacional Sajama (Bolivia) y ya se encuentra en contacto con la especie nativa B. funebris (Barahona \& Smith-Ramírez, datos sin publicar). Al mismo tiempo, afectaría servicios ecosistémicos de polinización locales generando perjuicios socio-ambientales y eventualmente políticos.

9.- Al CONTINUAR INGRESANDO ESPECIES EXoticas INVASORAS A NIVEL LATINOAMERICANO SE TIENEN LAS "MANOS ATADAS" PARA DESARROllar EFICIENTES MEDIDAS DE CONTROL. Un caso interesante de analizar 
Por qué Chile debe detener la importación de Bombus terrestris: Smith-RAmírez, C. ET AL.

es el de Japón, el cual comenzó el proceso de impedir la importación de B. terrestris en 2001. Esto se llevó a cabo a través de compromisos de responsabilidad social y empresarial de no ingreso de más abejorros comerciales, a menos que se requiera por motivos especiales muy justificados (Reade et al. 2016). A la vez, comenzó en forma paralela un proceso ciudadano de control, que para el año 2006 ya había eliminado poco más de 21.000 ejemplares (Horimoto et al. 2013). A través de estas eliminaciones los ciudadanos esperaban recuperar las poblaciones de sus abejorros nativos, como mostró Nagamitsu et al. (2010). Actualmente un nuevo método de control del abejorro comercial, basado en el uso de un insecticida, muestra ser altamente exitoso en la eliminación del invasor europeo (K. Goka, com. pers.). Este método ha sido desarrollado en Japón y está en fase de aplicarse masivamente en la isla Hokkaido. En el caso chileno pareciera sin sentido controlar una especie que se continua ingresando.

10.- Respeto a las etNias y su COSMOVisión. La posible extinción de $B$. dahlbomii, si no hay control de $B$. terrestris, puede producir no sólo una enorme pérdida en biodiversidad y servicio ecosistémico, sino en nuestra cultura. Bombus dahlbomii, conocido como dulliñ en mapudungun, representa el espíritu o la encarnación de los caciques y jefes de familia que vuelven a visitar a sus seres queridos cada primavera (Rosales 1674).

11.- Su ingreso y producción en Chile a partir de REINAS IMPORTADAS PODRÍA SER UN DELITO TANTO NACIONAL COMO INTERNACIONAL. El reglamento 291 presente en el Código Penal desde 1988 reza: "aquellos que ingresan indebidamente organismos, productos, elementos o agentes químicos, virales, bacteriológicos, radiactivos o de cualquier otro orden que por su naturaleza sean susceptibles de poner en peligro la salud animal o vegetal, o el abastecimiento de la población, serán penados con presidio menor en su grado máximo" (Bustos y Jaksic 2017). Debe analizarse cuidadosamente si en el caso del ingreso de $B$. terrestris a Chile se está infringiendo este reglamento o algún reglamento del Código Civil en relación a las obligaciones de los funcionarios del Estado. Actualmente, hay un proceso legal de investigación contra quien resulte responsable de la introducción de $B$. terrestris al país. Por otra parte, los reiterados hallazgos de enfermedades en B. terrestris (y en B. impatiens en otros países) procedentes de cultivo y vendidos con certificados libres de enfermedades, constituyen delitos comerciales y ambientales de índole internacional. Estos delitos debieran ser llevados por las autoridades sanitarias y ambientales de cada país a la
OMS (Organización Mundial de Comercio), CIADI (Centro Internacional de Arreglo de Diferencias) o INTERPOL (Organización Internacional de Policía Criminal), según corresponda. A su vez, Chile podría estar incurriendo en un delito internacional violando el tratado internacional con Argentina del año 1991, el cual trata sobre medioambiente.

Además, debe tenerse en consideración que:

AUn ES UN PROBLEMA A RESOLVER LA POLINIZACIÓN EN INVERNADERo a bajo costo. Creemos que existen varias soluciones alternativas al uso de $B$. terrestris en invernadero. Por ejemplo, en Argentina, una empresa que no obtuvo permiso de importación de $B$. terrestris desarrolló una tecnología para crianza de colmenas comerciales del abejorro nativo Bombus atratus (Franklin; Aizen et al. 2018). Cómo se señaló más arriba en Chile existen varias especies de abejas nativas zumbadoras posibles de usar en cultivos. Estas abejas podrían usarse cultivadas o usar las mismas que naturalmente existen. Atraer estas abejas nativas a los cultivos o invernaderos (semi abiertos) está mediado probablemente por la existencia de corredores de vegetación por los que puedan moverse y usar de hábitat. El uso de estas especies en invernaderos cerrados no ocurre por la falta de conocimiento y tecnología. En Chile, existe desde el 2013 una patente para criar colmenas del abejorro nativo $B$. dahlbomii en forma artificial (Estay 2013). Aunque hay una empresa que está trabajando para desarrollar el producto en forma comercial, en la actualidad aún no es posible adquirirlas. Sin embargo, es de señalar que al estar el abejorro nativo, $B$. dahlbomii en Peligro de Extinción producto, principalmente de la introducción del abejorro comercial (UICN 2015; MMA 2016) es importante cautelar la producción de colmenas comerciales aun cuando éstas sean producidas con especies nativas, ya que (1) en cautiverio tienen a proliferar enfermedades que luego son liberadas al ambiente siendo fácilmente transmitidas a otros individuos de la misma u otras especies, y (2) si su producción se basa en recolectar reinas silvestres estarán mermando poblaciones locales de la especie. Se recomienda promover la revegetación en alrededores de las áreas de cultivo para atraer a los polinizadores silvestres. Sin embargo, no debe descartarse el usar métodos complementarios como vibración que usan mano de obra, y por ende ofrecen además ventajas sociales en la generación de empleos.

El país debe trabajar en El DESARROLlo de UNA Ética AMBIENTAL, MAYOR SOSTENIBILIDAD, RESPONSABILIDAD SOCIAL Y EMPRESARIAL. Los empresarios y las autoridades sanitarias del país que usan o permiten el uso $B$. terrestris han actuado en desconocimiento de las consecuencias ambientales, sociales y económicas que ha tenido este hecho. Sin embargo, en el momento en que se tiene conocimiento existe un mal uso intencionado o dolo. El año 1998 y 1999 se le advirtió al SAG las consecuencias nefastas de su uso (Ruz 
2002). Posteriormente, durante el 2016 en adelante se le ha continuado advirtiendo al SAG a través de las Sociedades de Ecología y de Entomología de Chile, y en forma independiente, por expertos en polinización de Argentina y Chile. Sólo en el mes de abril del 2018, 22 medios de comunicación publicaron noticias advirtiendo sobre el daño a la biodiversidad producida por $B$. terrestris. Por lo demás, existen más de 90 trabajos científicos que desde 1996 en adelante tratan los problemas ocasionados por B. terrestris.

Las empresas que importan, crían y usan $B$. terrestris, y la autoridad sanitaria que permite su entrada al país, adolecen de una falta de responsabilidad social y ambiental que afecta a las actuales y futuras generaciones, existiendo razones éticas por las cuales su ganancia ocurre en desmedro del bien común y del patrimonio natural de Chile, y del resto de Sudamérica.

Ecológicamente, la sostenibilidad se describe cómo la permanencia de los sistemas biológicos a seguir siendo diversos y productivos a través del tiempo, condición necesaria para el bienestar humano y de otros organismos. Debe entenderse que la sostenibilidad, no es una moda, sino que una exigencia de los nuevos retos y reglas globales de competitividad que se están imponiendo en un entorno mundial de creciente demanda y escasez de los recursos naturales (Fernández \& Gutiérrez 2013).

REALIZAR UNA VALORACIÓN ECONÓMICA DEL INGRESO DE B. TERRESTRIS. Debe evaluarse si la ganancia de los productores que usan $B$. terrestris compensa y sobrepasa el daño económico ocasionado a la actividad melífera, a los huerteros, a los productores de frambuesa de Argentina y Chile, y quizás otros cultivos, a la pérdida de biodiversidad de estos países, y posible pérdida en Sudamérica, y a la alteración de las redes mutualistas planta-animal, con consecuencias en cadena que ni siquiera son posibles de visualizar.

Por las once razones expuestas, consideramos que el Estado debe velar y la ciudadanía debe exigir un consenso entre el fomento productivo y la conservación de las especies nativas y los procesos ecosistémicos en las cuales participan. Por su parte, el Estado, las empresas y la ciudadanía deben, además, proteger la producción de miel, la polinización realizada por la abeja melífera y los cultivos que son dañados por superabundancia de $B$. terrestris. Por lo cual, consideramos que:

a.- $\quad$ Chile debe detener inmediatamente las importaciones de $B$. terrestris para cultivos al aire libre, y comenzar a controlar esta especie donde esta tenga carácter invasora y no sea beneficiosa.

b.- Debe exigir compromiso empresarial ambiental, de forma que las colonias que ingresen desde fuera del país o sean producidas en este, sean liberadas sólo en invernaderos con cubierta adicional y doble puerta, y con permiso especial del Gobierno que no permita que escapen los abejorros (cómo sucede desde el 2006 en Japón). Además, debe garantizarse a la autoridad sanitaria que estos serán eliminados una vez usados. De no haber cumplimiento del compromiso el permiso a la empresa debiera caducar. A la vez, se debe implementar un sistema de control de cumplimiento. Los productores con invernadero que no cuenten con este sistema pueden volver al uso de hormonas, vibración o abrir los invernaderos (invernaderos semi abiertos) o buscar métodos alternativos. Estas alternativas, podrían producir menor productividad para los empresarios, aunque no necesariamente, pero si así lo fuera habría un mayor beneficio social y ambiental para el país.

c.- El gobierno debe exigir que las empresas que han comprado y aquellas que han sido parte de la cadena de adquisición y producción, que de aquí en adelante formen parte y se hagan cargo financieramente de los programas de control de la especie.

d.- Además, el Estado debe promover la investigación de modos alternativos de polinización en invernadero, de forma que se elimine la necesidad de usar $B$. terrestris.

e.- El Estado, a través del SAG, debe establecer un centro de pesquisa genética de forma que analice en sus propios laboratorios si los certificados "libre de enfermedades" que emiten las empresas exportadoras, independiente de la especie en cuestión, son ciertos o no. Es deber del SAG velar por la salud animal del país y, por lo tanto, hacer seguimiento de veracidad de estos certificados. Este seguimiento de veracidad debiera hacerse no sólo para $B$. terrestris, sino en general para todas las especies que ingresan, con o sin cuarentena. En el caso de que los certificados estén errados, se debe proceder a eliminar las muestras y hacer los descargos legales correspondientes a las autoridades sanitarias de los países exportadores que han faltado a la verdad.

f.- Finalmente, para el desarrollo de una agenda de trabajo y cumplimiento de compromisos, debe generarse una mesa conjunta de trabajo entre autoridades (Ministerio de Agricultura y de Medio Ambiente), empresarios, representantes de chacareros, científicos y ambientalistas. Esta mesa de trabajo se debiera incluir la participación de representantes de Argentina, dado los acuerdos sanitarios binacionales que probablemente no fueron respetados por Chile.

\section{AGRADECIMIENTOS}

Agradecemos al Dr. Marcelo Aizen por la revisión de este trabajo, apoyo en bibliografía de Marina Arbetman y el incentivo de Carolina Morales para escribirlo. Esta 
Por qué Chile debe detener la importación de Bombus terrestris: Smith-RAmírez, C. ET AL.

publicación es parte del proyecto SIGMA N`21565 MN/ UFRJ y la contribución número 24 del HYMN, Museu Nacional/Universidade Federal do Rio de Janeiro y del Programa API-3 de la Universidad de Los Lagos. Agradecimientos a CONICYT AFB 170008.

\section{REFERENCIAS}

Abak, K., Sari, N., Paksoy, M., Kaftanoglu, O., Yeninar, H. 1995. Efficiency of Bumblebees on the yield and quality of eggplant and tomato grown in unheated galsshouses. Acta Horticulturae 412: 268-274.

Abrahamovic, A.H., Díaz, N.B. 2002. Bumble bees of the Neotropical Region (Hymenoptera: Apidae). Biota Colombiana 3(2): 199-214.

AcostA, A.L. 2015. Will Bombus terrestris reach Brazil? A predictive study about a potential invasion. Tesis de Doctorado, Universidade de São Paulo, São Paulo, Brasil.

Acosta, A.L., Giannini, T.C., Imperatriz-Fonseca, V.L., Saraiva, A.M. 2016. Worldwide alien invasion: A methodological approach to forecast the potential spread of a highly invasive pollinator. PLoS one 11(2): e0148295.

Aizen, M.A., Smith-Ramírez, C., Morales, C.L., Vieli, L., Sáez, A., Barahona-Segovia, R.M., Arbetman, M., Montalva, J., Garibaldi, L. G., Inouye, D. W., Harder, L.D. 2018. Conflicting importation policies foster a massive bumble-bee invasion in South America, Lack of international coordination on species trade has detrimental environmental consequences. Journal of Applied Ecology. 2018;00:1-7. doi: 10.1111/1365-2664.13121

Aouar-Sadli, M., Louadi, K., Doumandi, S.E. 2008. Pollination of the broad bean (Vicia faba L. var. major) (Fabaceae) by wild bees and honey bees (Hymenoptera: Apoidea) and its impact on the seed production in the Tizi-Ouzou area (Algeria). African Journal of Agricultural Research 3(4): 266-272.

Bustos, T., JAKsic, F. 2017. Marco regulatorio aplicable al proceso de internación de especies exóticas a la República de Chile. Ediciones CAPES-UC, Santiago, Chile.

Dafni, A., Shmida, A. 1996. The possible ecological implications of the invasion of.Bombus terrestris. In: Matheson, A., Buchmann, S.L., O’Toole, C., Westrich, P., Williams, I.H. (eds.). The Conservation of Bees: 183-200. Academic Press.

Dafni, A., Kevan, P., Gross, C.L., Goka, K. 2010. Bombus terrestris, pollinator invasive and pest: An assessment of problems associated with its widespread introductions for commercial purposes. Applied Entomology and Zoology 45(1): 101-113.

Dasgan, H.Y., Özdo/an, A.O., KaftandLu, O., AbaK, K. 2004. Effectiveness of bumblebee pollination in anti-frost heated tomato greenhouse in the mediterranean basin. Turkish Journal of Agriculture and Forestry 28 (2): 73-82.

Dohzono, I., Kufnitake, Y.K., Yokoyama, J., GoKa, K. 2008. Alien bumble bee affects native plant reproduction through interactions with native bumble bees. Ecology 89(11): 3082-3092.

Estay, P. 2007. Bombus en Chile: especies, biología y manejo.
Colección Num. 22. Instituto de Investigaciones Agrarias (INIA), Santiago, Chile.

Estay, P. 2013. Método artificial de domesticación y crianza de Bombus dahlbomii que comprende recolectar material parental de reinas, seleccionar reinas fecundadas con un peso de 0,96-1,54 g, alimentar las reinas, obtener obreras e instalar las colmenas en el terreno definitivo a polinizar. Chile, 49670 (A01G1/04, A01K47/00). 4/12/2013. Appl. 15/07/2005.

Fernández, L., Gutiérrez, M. 2013. Bienestar Social, Económico y Ambiental para las Presentes y Futuras Generaciones. Innovacion Tecnologica 24(2): 121-130.

Genersch, E., Yue, C., Fries, I., DE Miranda, J.R. 2006. Detection of Deformed wing virus, a honey bee viral pathogen, in bumble bees (Bombus terrestris and Bombus pascuorum) with wing deformities. Journal of invertebrate pathology 91(1): 61-63.

Graham, M. 2015. Efecto del robo de néctar por el abejorro invasor Bombus terrestris sobre la polinización y el éxito reproductivo de la arvejilla nativa Vicia nigricans (Fabaceae). Tesis Licenciatura en Biología, Universidad de Comahue, Bariloche, Argentina.

Graystock, P., Yates, K., Evison, S.E.F., Darvill, B., Goulson, D., Hughes, W.H.O. 2013. The Trojan hives: Pollinator pathogens, imported and distributed in bumblebee colonies. Journal of Applied Ecology 50: 1207-1215.

Hingston, A.B. 2006. Is the exotic bumblebee Bombus terrestris really invading Tasmanian native vegetation? Journal of Insect Conservation 10(3): 289-293.

Hingston, A.B., McQuillan, P.B. 1998. Does the recently introduced bumblebee Bombus terrestris (Apidae) threaten Australian ecosystems? Austral Journal of Ecology 23: 539-549.

Hogendoorn, K., Steen, Z., SchwarZ, M.P. 2000. Native Australian carpenter bees as a potential alternative to introducing bumblebees for tomato pollination in greenhouses. Journal of Apicultural Research 39 (1-2): 67-74.

Horimoto, R., Noriko, K., Izumi, W. 2013. Measures against an invasive alien species, Bombus terrestris, using a participatory monitoring program involving continuous participation and information transmission. Japanese Journal of Conservation Ecology 18: 213-224.

Inari, N., Nagamitsu, T., Kenta, T., Goka, K., Hiura, T. 2005. Spatial and temporal pattern of introduced Bombus terrestris abundance in Hokkaido, Japan, and its potential impact on native bumblebees. Population Ecology 47: 7782.

Inoue, M.N., Yokoyama, J., Washitani, I. 2008. Displacement of Japanese native bumblebees by the recently introduced Bombus terrestris (L.) (Hymenoptera: Apidae). Journal of Insect Conservation 12(2): 135-146.

Kendall, D.A., Smith, B.D. 1976. The Pollinating Efficiency of Honeybee and Bumblebee Visits to Flowers of the Runner bean Phaseolus coccineus L. Journal of Applied Ecology: 749-752.

Kenis, M., Auger-Rozenberg, M.A., Roques, A., Timms, L., Péré, C., Cock, M.J.W., Settele, J., Augustin, S., LopezVAAmonde, C. 2008. Ecological effects of invasive alien insects. Biological Invasions 11:21-45.

Kenta, T., Inari, N., Nagamitsu, T., Goka, K., Hiura, T. 2007. 
Commercialized European bumblebee can cause pollination disturbance: An experiment on seven native plant species in Japan. Biological Conservation 134(3):298-309. doi: 10.1016/j.biocon.2006.07.023.

LIETEN. 1993. Vruchtmisvorming bij aardbeien: overbevlieging F. LIETËN, Proefbedrijf der Noorderkempen Meerle. FRUITTEELT-Berichten: 27-29.

Medel, R., González-Browne, C., Salazar, D., Ferrer, P., EHRENFELD, M. 2018. The most effective pollinator principle applies to new invasive pollinators. Biology Letters 14(6):20180132. doi: 10.1098/rsbl.2018.0132.

Meeus, I., Brown, M.J.F., De GraAf, D.C., Smagghe, G. 2011. Effects of invasive parasites on bumble bee declines. Conservation Biology 25:662-671

MMA. 2016. Ficha de Antecedentes de especie. Bombus dahlbomii. Ministerio de Medio Ambiente. URL:http:// www.mma.gob.cl/clasificacionespecies/fichas12proceso/ pac/Bombus_dahlbomii_12RCE_INICIO.pdf. Accedido: Enero 15, 2018.

Montalva, J., Sepúlveda, V., Vivallo, F., Silva, D.P. 2017. New records of an invasive bumble bee in northern Chile: expansion of its range or new introduction events? Journal of Insect Conservation 21:657-666.

Morales, C.L., Arbetman, M.P., Cameron, S.A., Aizen, M.A. 2013. Rapid ecological replacement of a native bumble bee by invasive species. Frontiers in Ecology and the Environment 11: 529-534. https://doi. org/10.1890/120321.

Morales, C.L., Sáez, A., Garibaldi, L.A., Aizen, M.A. 2017. Disruption of pollination services by invasive pollinator species. Vila, M., Hulme, P., Ruiz, G. (Eds.), Impact of biological invasions on ecosystem services (pp. 203220). Dordrecht, The Netherlands: Springer. https://doi. org/10.1007/978-3-319-45121-3.

Murray, T.E., Coffey, M.F., Kehoe, E., Horgan, F.G. 2013. Pathogen prevalence in commercially reared bumble bees and evidence of spillover in conspecific populations. Biological Conservation 159: 269-276.

Nagamitsu, T., Yamagishi, H., Kenta, T., Inari, N., Kato, E. 2010. Competitive effects of the exotic Bombusterrestris on native bumble bees revealed by a field removal experiment. Popultion Ecology 52(1):123-136. doi: 10.1007/s10144009-0151-7.

ODEPA. 2013. Situación del tomate para consumo fresco. URL:http://www.odepa.gob.cl/odepaweb/publicaciones/ doc/11729.pdf. Accedido: Enero 15, 2018.

Potts, S.G., Imperatriz-Fonseca, V.L., NGo, H.T., Biesmeijer, J.C., Breeze, T.D., Dicks, L.V., Garibaldi, L.A., Hill, R., Settele, J., Vanbergen, A.J., Aizen, M.A., Cunningham, S.A., Eardley, C., Freitas, B.M., Gallai, N., Kevan, P.G., Kovács-Hostyánszki, A., Kwapong, P.K., Li, J., Li, X., Martins, D.J., Nates-Parra, G., Pettis, J.S., Rader, R., VIANA, V.F.(Eds.).2016.IPBES: Summary for policymakers of the assessment report of the Intergovernmental Science-Policy Platform on Biodiversity and Ecosystem Services on pollinators, pollination and food production. Report. Intergovernmental Science-Policy Platform on Biodiversity and Ecosystem Services, Bonn, Germany.

Rasmussen, C. 2003. Clave de identificación para las especies peruanas de Bombus Latretille, 1809 (Hymenoptera, Apidae), con notas de su biología y distribución. Revista
Peruana de Entomología 43:31-45.

Reade, C., Goka, K., Thorp, R., Mitsuhata, M., Wasbauer, M. 2016. From corporate social responsibility to accountability in the bumblebee trade: A Japanese perspective. En: Atkins, J., Atkins, B. (Eds.) The business of bees: An integrated approach to bee decline and corporate responsibility. 6988 pp. Greenleaf Publishing. Yorkshire, UK. https://doi. org/10.9774/GLEAF.9781783534340_5.

Rosales, D. 1674. Publicado1877. Historia General del Reyno de Chile. Flandes Indiano, 3.

RuZ, L. 2002. Bee pollinators introduced to Chile: a review. En: Kevan, P., Imperatriz, F. (Eds) Pollinating bees - the conservation link between agriculture and nature. Ministry of Environment. Brasília, Brazil.

Sachman-Ruiz, B., Narváez-Padilla, V., Reynaud, E. 2015. Commercial Bombus impatiens as reservoirs of emerging infectious diseases in central México. Biological Invasions 17: 2043-2053.

Sáez, A., Morales, C.L., Ramos, L.Y., Aizen, M.A. 2014. Extremely frequent bee visits increase pollen deposition but reduce drupelet set in raspberry. Journal Applied Ecology 51:1603-1612. doi: 10.1111/1365-2664.12325.

Sáez, A., Moralesa, C.L., Garibaldib, L.A., Aizen, M.A. 2017. Invasive bumble bees reduce nectar availability for honey bees by robbing raspberry flower buds. Basic and Applied Ecology 19: 26-35.

SAG. 2016. Servicio Agrícola Ganadero of Chile. Data provided upon request in June 2016 according to the law of "Transparency and Access to Public Information" (Reference AR006T0000668), Santiago, Chile.

SAG. 2017. Servicio Agrícola Ganadero of Chile. Data provided upon request in June 2016 according to the law of "Transparency and Access to Public Information" (Reference AR006T0001145), Santiago, Chile.

Schmid-Hempel, R., Eckhardt, M., Goulson, D., Heinzmann, D., Lange, C., Plischuk, S., Escudero, L.R., Salathe, R., Scriven, J.J., Schmid-Hempel, P. 2014. The invasion of the southern South America by imported bumblebees and associated parasites. Journal of Animal Ecology 83: 823-837.

Simberloff, D., Martin, J.L., Genovesi, P., Maris, V., Wardle, D.A., Aronson, J., Courchamp, F., Galil, G., GarcíaBerthou, E., Pascal, M., Pyšek, P. 2013. Impacts of biological invasions: what's what and the way forward? Trends in Ecology \& Evolution, 28(1): 58-66.

Singh, R., Levitt, A.L., Rajotte, E.G., Holmes, E.C., Ostiguy, N. 2010. RNA viruses in hymenopteran pollinators: evidence of inter-taxa virus transmission via pollen and potential impact on non-Apis hymenopteran species. PLoS ONE $5: 1-16$

Smith-Ramírez, C., Ramos-Jiliberto, C., Valdovinos, F., Martínez, P., Castillo, J., Armesto, J. 2014. One decade of changes in the pollinator assemblage of the temperate tree Eucryphia cordifolia (Cunoniaceae): nested community structure. Oecologia 176: 156-169.

Tehel, A., Brown, M.J., Paxton, R.J. 2016. Impact of managed honey bee viruses on wild bees. Current opinion in virology 19: 16-22.

Trillo, A., Herrera, J., Vilà M. 2018. Managed bumble bees increase flower visitation but not fruit weight in polytunnel 
Por qué Chile debe detener la importación de Bombus terrestris: Smith-RAmírez, C. ET AL.

strawberry crops. Basic and Applied Ecology 30: 32-40.

UICN. 2015. Threatened List of Endangered Species. Bombus dahlbomii. URL:http://www.iucnredlist.org/ details/21215142/0. Accedido: Enero 15, 2018.

Valdivia, C.E., Carroza, J.P., Orellana, J.I. 2016. Geographic distribution and trait-mediated causes of nectar robbing by the European bumblebee Bombus terrestris on the Patagonian shrub Fuchsia magellanica. Flora Morphology, Distribution, Functional Ecology of Plants 225: $30-36$

Valdivia, C.E., Orellana, J.I., Morales-Paredes, C. 2018. Ant-mediated nectar robbing from the Chilean firetree Embothrium coccineum (Proteaceae): no effect on seed production. Annales Botanici Fennici 55: 217-226.

VAN RAVESTIJN, W., Steen, J. VAN DER. 1991. Use of bumblebees for the pollination of glasshouse tomatoes. Acta Horticulturae, 288: 204-212.

Velthius, H.W., Van Doorn, A. 2006. A century of advances in bumblebee domestication and the economic and environmental aspects of its commercialization for pollination. Apidologie 37:421-451

Vieli, L., Davis, F.W., Kendall, B.E., Altieri, M. 2016. Landscape effects on wild Bombus terrestris queens visiting high bush blueberry fields in south-central Chile. Apidologie, 47(5): 711-716. doi: 10.1007/s13592-015-0422-6.

Winter, K., Adams, L., Thorp, R., Inouye, D., Day, L., Ascher, J., Buchmann, S. 2006. Importation of non-native Bumble bees into North America: potential consequences of using Bombus terrestris and other non-native bumblebees for greenhouse crop pollination in Canada, Mexico, and the United States. White Paper of the North American Pollinator Protection Campaign. USA.

Zisovich, A.H., Goldway, M., Schneider, D., Steinberg, S., Stern, E., Stern, R.A. 2012. Adding bumblebees (Bombus terrestris L., Hymenoptera: Apidae) to pear orchards increases seed number per fruit, fruit set, fruit size and yield. The Journal of Horticultural Science and Biotechnology 87(4):353-359.

Recibido: 18.06.2018

Aceptado: 03.08.2018 